\title{
Negativity and strong monogamy of multi-party quantum entanglement beyond qubits
}

\author{
Jin Hyuk Choi ${ }^{1}$ and Jeong San $\mathrm{Kim}^{2}$, * \\ 1 Humanitas College, Kyung Hee University, Yongin-si, Gyeonggi-do 446-701, Korea \\ 2 Department of Applied Mathematics and Institute of Natural Sciences, \\ Kyung Hee University, Yongin-si, Gyeonggi-do 446-701, Korea
}

(Dated: October 6, 2018)

\begin{abstract}
We propose the square of convex-roof extended negativity(SCREN) as a powerful candidate to characterize strong monogamy of multi-party quantum entanglement. We first provide a strong monogamy inequality of multi-party entanglement using SCREN, and show that the tangle-based multi-qubit strong monogamy inequality can be rephrased by SCREN. We further show that SCREN strong monogamy inequality is still true for the counterexamples that violate tangle-based strong monogamy inequality in higher-dimensional quantum systems rather than qubits. We also analytically show that SCREN strong monogamy inequality is true for a large class of multi-qudit states, a superposition of multi-qudit generalized W-class states and vacuums. Thus SCREN is a good alternative to characterize the strong monogamy of entanglement even in multi-qudit systems.
\end{abstract}

PACS numbers: $03.67 . \mathrm{Mn}$, 03.65.Ud

\section{INTRODUCTION}

Quantum entanglement is a quantum correlation used as a resource in various applications of quantum information theory such as quantum teleportation and quantum cryptography [1 3]. One important property of entanglement is its restricted shareability in multi-party quantum systems, which does not have any classical counterpart. This restriction of entanglement shareability among multi-party systems is known as the monogamy of entanglement (MoE) [4-8].

The first mathematical characterization of MoE was established by Coffman-Kundu-Wootters(CKW) as an inequality [4]; for a three-qubit pure state $|\psi\rangle_{A B C}$,

$$
\tau\left(|\psi\rangle_{A \mid B C}\right) \geq \tau\left(\rho_{A \mid B}\right)+\tau\left(\rho_{A \mid C}\right)
$$

where $\tau\left(|\psi\rangle_{A \mid B C}\right)$ is the one-tangle of $|\psi\rangle_{A B C}$ quantifying the pure state entanglement between $A$ and $B C$, and $\tau\left(\rho_{A \mid B}\right)$ (similarly with $\left.\tau\left(\rho_{A \mid C}\right)\right)$ is the two-tangle of the reduced density matrix $\rho_{A B}=\operatorname{tr}_{C}|\psi\rangle_{A B C}\langle\psi|$ quantifying the two-qubit entanglement inherent in $\rho_{A B}$.

This inequality is also referred to as $C K W$ inequality, and it shows the mutually exclusive nature of two-qubit entanglement shared in three-qubit systems; more entanglement shared between two qubits $A$ and $B$ leads to less entanglement between the other two qubits $A$ and $C$ so that their summation does not exceed the total entanglement between $A$ and $B C$. Moreover, the residual entanglement from the difference between left and right-hand sides of $\mathrm{CKW}$ inequality is interpreted as the genuine three-qubit entanglement, three-tangle.

Later, CKW inequality was generalized for multi-qubit systems [5] as well as some cases of higher-dimensional

*Electronic address: freddie1@khu.ac.kr quantum systems [9-12]. A general monogamy inequality for arbitrary quantum systems was established in terms of the squashed entanglement [13, 14].

Recently, the definition of three-tangle was generalized into arbitrary $n$-qubit systems, namely $n$-tangle quantifying the genuine multi-qubit entanglement. By conjecturing the nonnegativity of the $n$-tangle, the concept of strong monogamy(SM) inequality of $n$-qubit entanglement was proposed [15]. Although an analytical proof of SM conjecture for arbitrary multi-qubit states seems to be a formidable challenge due to the numerous optimization processes arising in the definition of $n$-tangle, an extensive numerical evidence was presented for four qubit systems together with an analytical proof for some cases of multi-qubit systems [15, 16].

However, tangle is known to fail in the generalization of CKW inequality for higher dimensional quantum systems rather than qubits; there exist quantum states in $3 \otimes 3 \otimes 3$ and even in $3 \otimes 2 \otimes 2$ quantum systems violating CKW inequality [17, 18]. Because SM inequality proposed in [15] is reduced to CKW inequality for $n=3$, these counterexamples of CKW inequality also implies the violation of SM inequality using tangles in higherdimensional systems rather than qubits.

Here we propose the square of convex-roof extended negativity(SCREN) as a powerful candidate to characterize the strongly monogamous property of multi-qudit systems. We first provide a SM inequality of multi-party entanglement using SCREN, and show that the SM inequality of multi-qubit entanglement using tangle [15, 16. can be rephrased by SCREN. This SCREN SM inequality is also true for the counterexamples of tangle in higherdimensional systems. Moreover, we analytically show that SCREN SM inequality is saturated by a large class of multi-qudit states, a superposition of multi-qudit generalized W-class states and vacuums. Thus SCREN is a good alternative for strong monogamy of multi-party entanglement even in higher-dimensional systems.

The paper is organized as follows. In Sec. IIA, we re- 
view the definition of negativity, and provide the relation between tangle and SCREN for multi-qubit monogamy inequality in Sec. IIB, In Sec. IIIA, we recall the multiqubit SM inequality in terms of tangle, and propose a multi-qudit SM inequality using SCREN in IIIB In Sec. IVA, we provide the definition of multi-qudit generalized W-class states as well as some useful properties of this class of states. In Sec. IVB, we analytically show that the SCREN SM inequality of multi-qudit entanglement is saturated by a superposition of generalized $\mathrm{W}$ class states and vacuum. In Sec. $\mathrm{V}$. we summarize our results.

\section{NEGATIVITY AND MONOGAMY OF MULTI-PARTY QUANTUM ENTANGLEMENT}

\section{A. Negativity}

For a bipartite pure state $|\phi\rangle_{A B}$ in a $d \otimes d^{\prime}\left(d \leq d^{\prime}\right)$ quantum system with its Schmidt decomposition,

$$
|\phi\rangle_{A B}=\sum_{i=0}^{d-1} \sqrt{\lambda_{i}}|i i\rangle, \quad \lambda_{i} \geq 0, \quad \sum_{i=0}^{d-1} \lambda_{i}=1,
$$

its negativity is defined as

$$
\mathcal{N}\left(|\phi\rangle_{A \mid B}\right)=\||\phi\rangle_{A B}\left\langle\left.\phi\right|^{T_{B}} \|_{1}-1=2 \sum_{i<j} \sqrt{\lambda_{i} \lambda_{j}},\right.
$$

where

$$
|\phi\rangle_{A B}\left\langle\left.\phi\right|^{T_{B}}=\sum_{i, j=0}^{d-1} \sqrt{\lambda_{i} \lambda_{j}} \mid i j\right\rangle_{A B}\langle j i|
$$

is the partial transposition of $|\phi\rangle_{A B}$ and $\|\cdot\|_{1}$ is the trace norm [19].

Because the possible negative eigenvalues of the partially transposed state in Eq. (3) are $-\sqrt{\lambda_{i} \lambda_{j}}$ for $i<j$ with corresponding eigenstates $\left|\psi_{i j}\right\rangle_{A B}=\frac{1}{\sqrt{2}}\left(|i j\rangle_{A B}-\right.$ $|j i\rangle_{A B}$ ), the definition of negativity in Eq. (2) is thus the sum of all possible negative eigenvalues with a constant proportion [20]. Eq. (22) can also have an alternative definition as

$$
\mathcal{N}\left(|\phi\rangle_{A \mid B}\right)=2 \sum_{i<j} \sqrt{\lambda_{i} \lambda_{j}}=\left(\operatorname{tr} \sqrt{\rho_{A}}\right)^{2}-1,
$$

where $\rho_{A}=\operatorname{tr}_{B}|\phi\rangle_{A B}\langle\phi|$ is the reduced density matrix of $|\phi\rangle_{A B}$ on subsystem $A$. For a bipartite mixed state $\rho_{A B}$, its negativity is analogously defined as

$$
\mathcal{N}\left(\rho_{A \mid B}\right)=\left\|\rho_{A B}{ }^{T_{B}}\right\|_{1}-1
$$

where $\rho_{A B}^{T_{B}}$ is the partial transposition of $\rho_{A B}$.

Positive partial transposition(PPT) [21, 22] gives a separability criterion for bipartite pure states and two-qubit mixed states. PPT is also a necessary and sufficient condition for nondistillability in $2 \otimes n$ quantum system [23, 24]. However, there also exist entangled mixed states with $\mathrm{PPT}$ in higher-dimensional quantum systems rather than $2 \otimes 2$ or $2 \otimes 3$ quantum systems. [23, 25]. For this case, negativity in Eq. (5) cannot distinguish PPT bound entangled states from separable states, and thus, negativity itself is not sufficient to be a good measure of entanglement even in a $2 \otimes n$ quantum system.

One way to overcome this rack of separability criterion of negativity in higher-dimensional mixed quantum states is using convex-roof extension [26]; for a bipartite mixed state mixed state $\rho_{A B}$, its convex-roof extended negativity is

$$
\mathcal{N}_{m}\left(\rho_{A \mid B}\right)=\min _{\left\{p_{k},\left|\phi_{k}\right\rangle\right\}} \sum_{k} p_{k} \mathcal{N}\left(\left|\phi_{k}\right\rangle_{A \mid B}\right),
$$

where the minimum is taken over all possible pure state decompositions of $\rho_{A B}=\sum_{k} p_{k}\left|\phi_{k}\right\rangle_{A B}\left\langle\phi_{k}\right|$. Convexroof extended negativity gives a perfect discrimination of PPT bound entangled states and separable states in any bipartite quantum system. Moreover, it was also shown that the quantity in Eq. (6) cannot be increased by local quantum operations and classical communications(LOCC) [9, 26].

\section{B. Monogamy Inequality Using Negativity}

For a two-qubit pure state $|\psi\rangle_{A B}$ [27], its tangle (or one-tangle) is defined as

$$
\tau\left(|\psi\rangle_{A \mid B}\right)=4 \operatorname{det} \rho_{A},
$$

with the reduced density matrix $\rho_{A}=\operatorname{tr}_{B}|\psi\rangle_{A B}\langle\psi|$. For a two-qubit mixed state $\rho_{A B}$, its tangle (or two-tangle) is defined as

$$
\tau\left(\rho_{A \mid B}\right)=\left[\min _{\left\{p_{h},\left|\psi_{h}\right\rangle\right\}} \sum_{h} p_{h} \sqrt{\tau\left(\left|\psi_{h}\right\rangle_{A \mid B}\right)}\right]^{2} .
$$

where the minimization is taken over all possible pure state decompositions

$$
\rho_{A B}=\sum_{h} p_{h}\left|\psi_{h}\right\rangle_{A B}\left\langle\psi_{h}\right| .
$$

Mathematically, monogamy of multi-party quantum entanglement was first characterized in three-qubit systems by Coffman, Kundu and Wootters(CKW) [4]; using one and two tangles as the bipartite entanglement quantification, monogamy inequality of three-qubit entanglement was proposed as

$$
\tau\left(|\psi\rangle_{A \mid B C}\right) \geq \tau\left(\rho_{A \mid B}\right)+\tau\left(\rho_{A \mid C}\right),
$$

where $\tau\left(|\psi\rangle_{A \mid B C}\right)$ is the one tangle of the three-qubit pure state $|\psi\rangle_{A B C}$ quantifying the bipartite entanglement between $A$ and $B C$, and $\tau\left(\rho_{A \mid B}\right)$ and $\tau\left(\rho_{A \mid C}\right)$ are 
the two tangles of the two-qubit reduced states $\rho_{A B}=$ $\operatorname{tr}_{C}|\psi\rangle_{A B C}\langle\psi|$ and $\rho_{A C}=\operatorname{tr}_{B}|\psi\rangle_{A B C}\langle\psi|$, respectively.

Later CKW inequality in (10) was generalized into $n$ qubit systems [5] as

$$
\tau\left(|\psi\rangle_{A_{1} \mid A_{2} \cdots A_{n}}\right) \geq \sum_{j=2}^{n} \tau\left(\rho_{A_{1} \mid A_{j}}\right)
$$

for one tangle $\tau\left(|\psi\rangle_{A_{1} \mid A_{2} \cdots A_{n}}\right)$ and two tangles $\tau\left(\rho_{A_{1} \mid A_{j}}\right)$ of each two-qubit reduced density matrices $\rho_{A_{1} A_{j}}$ on subsystems $A_{1} A_{j}$ for each $j=2, \cdots, n$. However, tangle is known to fail in the generalization of CKW inequality for higher dimensional quantum systems rather than qubits; there exist quantum states in $3 \otimes 3 \otimes 3$ and even in $3 \otimes 2 \otimes 2$ quantum systems violating CKW inequality in (10) 17, 18].

Now we consider another generalization of tangles from qubits to qudit systems using negativity [9]. We first note that for any pure state $|\psi\rangle_{A B}$ with Schmidt-rank 2 (especially for two-qubit pure state)

$$
|\psi\rangle_{A B}=\sqrt{\lambda_{1}}\left|e_{0}\right\rangle_{A} \otimes\left|f_{0}\right\rangle_{B}+\sqrt{\lambda_{2}}\left|e_{1}\right\rangle_{A} \otimes\left|f_{1}\right\rangle_{B},
$$

the square of negativity in Eq. (4) coincides with the tangle in Eq. (7)

$$
\mathcal{N}^{2}\left(|\psi\rangle_{A \mid B}\right)=4 \lambda_{1} \lambda_{2}=\tau\left(|\psi\rangle_{A \mid B}\right) .
$$

Thus the two-tangle of any two-qubit state $\rho_{A B}$ in Eq. (8) can be rephrased as

$$
\tau\left(\rho_{A \mid B}\right)=\left[\min _{\left\{p_{h},\left|\psi_{h}\right\rangle\right\}} \sum_{h} p_{h} \mathcal{N}\left(\left|\psi_{h}\right\rangle_{A \mid B}\right)\right]^{2}
$$

where the right-hand side of Eq. (14) is square of the convex-roof extended negativity in Eq. (6). Based on this idea, we propose a bipartite entanglement measure using negativity; for any two-qudit mixed state $\rho_{A B}$ its square of convex-roof extended negativity(SCREN) is defined as

$$
\mathcal{N}_{s c}\left(\rho_{A \mid B}\right)=\left[\min _{\left\{p_{h},\left|\psi_{h}\right\rangle\right\}} \sum_{h} p_{h} \mathcal{N}\left(\left|\psi_{h}\right\rangle_{A \mid B}\right)\right]^{2}
$$

From the properties of convex-roof extended negativity in Eq. (6), it is straightforward to check that SCREN has the separability criterion and monotonicity under LOCC [26]. We also note that Eqs. (14) and (15) imply the coincidence of SCREN with two-tangle for any two-qubit state $\rho_{A B}$,

$$
\mathcal{N}_{s c}\left(\rho_{A \mid B}\right)=\tau\left(\rho_{A \mid B}\right)
$$

Consequently, the multi-qubit monogamy inequality in terms of tangles in (11) can be rephrased in terms of SCREN as,

$$
\mathcal{N}_{s c}\left(|\psi\rangle_{A_{1} \mid A_{2} \cdots A_{n}}\right) \geq \sum_{j=2}^{n} \mathcal{N}_{s c}\left(\rho_{A_{1} \mid A_{j}}\right) .
$$

Moreover, Inequality (17) still holds for the counterexamples [17, 18] that violate CKW inequality in higherdimensional systems [9]. Thus SCREN is a good generalization of two-qubit tangle into higher-dimensional quantum systems without any known counterexamples even in higher-dimensional quantum systems so far.

\section{STRONG MONOGAMY OF MULTI-PARTY QUANTUM ENTANGLEMENT}

\section{A. Multi-Qubit Strong Monogamy Inequality}

For any three-qubit pure state $|\psi\rangle_{A B C}$, the residual entanglement from the difference between left and righthand sides of CKW Inequality (10) is also interpreted as the genuine three-party entanglement, namely threetangle of $|\psi\rangle_{A B C}$

$$
\tau\left(|\psi\rangle_{A|B| C}\right)=\tau\left(|\psi\rangle_{A \mid B C}\right)-\tau\left(\rho_{A \mid B}\right)-\tau\left(\rho_{A \mid C}\right)
$$

The three-tangle in Eq. (18) is a good measure of genuine three-qubit entanglement, which is invariant under the permutation of subsystems $A, B$ and $C$ [28].

The definition of three-tangle was generalized for arbitrary $n$-qubit quantum states [15]; for an $n$-qubit pure state $|\psi\rangle_{A_{1} A_{2} \cdots A_{n}}$, its $n$-tangle is defined as

$$
\begin{aligned}
\tau\left(|\psi\rangle_{A_{1}\left|A_{2}\right| \cdots \mid A_{n}}\right)= & \tau\left(|\psi\rangle_{A_{1} \mid A_{2} \cdots A_{n}}\right) \\
& -\sum_{m=2}^{n-1} \sum_{\vec{j}^{m}} \tau\left(\rho_{A_{1}\left|A_{j_{1}^{m}}\right| \cdots \mid A_{j_{m-1}^{m}}}\right)^{m / 2},
\end{aligned}
$$

where the index vector $\vec{j}^{m}=\left(j_{1}^{m}, \ldots, j_{m-1}^{m}\right)$ spans all the ordered subsets of the index set $\{2, \ldots, n\}$ with $(m-1)$ distinct elements. Eq. (19) is a recurrent definition that needs all the $m$ tangles $\tau\left(\rho_{A_{1}\left|A_{j_{1}^{m}}\right| \cdots \mid A_{j_{m-1}^{m}}}\right)$ of $m$-qubit reduced density matrices $\rho_{A_{1} A_{j_{1}^{m}} \cdots A_{j_{m-1}^{m}}}$ for $2 \leq m \leq$ $n-1$, where $\tau\left(\rho_{A_{1}\left|A_{j_{1}^{m}}\right| \cdots \mid A_{j_{m-1}^{m}}}\right)$ is defined as 


$$
\tau\left(\rho_{A_{1}\left|A_{j_{1}^{m}}\right| \cdots \mid A_{j_{m-1}^{m}}}\right)=\left[\min _{\left\{p_{h},\left|\psi_{h}\right\rangle\right\}} \sum_{h} p_{h} \sqrt{\tau\left(\left|\psi_{h}\right\rangle_{\left.A_{1} \mid A_{j_{1}^{m}|\cdots| A_{j_{m-1}^{m}}}\right)}\right)}\right]^{2}
$$

with the minimization over all possible pure state decompositions

$$
\rho_{A_{1} A_{j_{1}^{m}} \cdots A_{j_{m-1}^{m}}}=\sum_{h} p_{h}\left|\psi_{h}\right\rangle_{A_{1} A_{j_{1}^{m} \cdots A_{j_{m-1}^{m}}}}\left\langle\psi_{h}\right| .
$$

For $n=3$, the definition of $n$-tangle in Eq. (19) reduces to that of three-tangle in Eq. (18) whose nonnegativity is equivalent to the CKW inequality (10). In other words, the nonnegativity of three-tangle provides us with a quantitative characterization of three-qubit monogamy of entanglement. For $n=2$, Eq. (20) also reduces to the two-tangle of two-qubit state $\rho_{A_{1} A_{2}}$ in Eq. (8).

Based on this idea, a strong monogamy(SM) inequality of multi-qubit entanglement was proposed as

$$
\tau\left(|\psi\rangle_{A_{1} \mid A_{2} \cdots A_{n}}\right) \geq \sum_{m=2}^{n-1} \sum_{\vec{j}^{m}} \tau\left(\rho_{A_{1}\left|A_{j_{1}^{m}}\right| \cdots \mid A_{j_{m-1}^{m}}}\right)^{m / 2}
$$

by conjecturing the nonnegativity of $n$-tangle in Eq. (19). The lower term in Inequality (22) appears in between the both sides of the $n$-qubit CKW inequality in (11) as

$$
\begin{aligned}
\tau\left(|\psi\rangle_{A_{1} \mid A_{2} \cdots A_{n}}\right) \geq & \sum_{j=2}^{n} \tau\left(\rho_{A_{1} \mid A_{j}}\right) \\
& +\sum_{m=3}^{n-1} \sum_{\vec{j}^{m}} \tau\left(\rho_{A_{1}\left|A_{j_{1}^{m}}\right| \cdots \mid A_{j_{m-1}^{m}}}\right)^{m / 2} \\
\geq & \sum_{j=2}^{n} \tau\left(\rho_{A_{1} \mid A_{j}}\right),
\end{aligned}
$$

therefore it is a stronger inequality. We also note that Inequality (22) encapsulates three-qubit CKW inequality in (10) for $n=3$. Thus Inequality (22) is another generalization of three-qubit monogamy inequality into multi-qubit systems in a stronger form.

For the validity of SM inequality in (22), an extensive numerical evidence was presented for four qubit systems together with analytical proof for some cases of multiqubit systems. It was also recently shown that Inequality (22) is also true for a large class of multi-qubit generalized W-class states,

$$
\begin{aligned}
|\psi\rangle_{A_{1} A_{2} \ldots A_{n}}= & a_{1}|10 \cdots 0\rangle+a_{2}|01 \cdots 0\rangle \\
& +\ldots+a_{n}|00 \cdots 1\rangle
\end{aligned}
$$

with $\sum_{i=1}^{n}\left|a_{j}\right|^{2}=1$ [16].

\section{B. SCREN Strong Monogamy Inequality}

Although Inequality (22) proposes a stronger monogamous property of multi-qubit entanglement with various cases of analytic proof, Inequality (22) is no longer valid for higher-dimensional quantum systems rather than qubits; for $n=3$, Inequality (22) becomes a CKWtype inequality of three-party quantum systems,

$$
\tau\left(|\psi\rangle_{A \mid B C}\right) \geq \tau\left(\rho_{A \mid B}\right)+\tau\left(\rho_{A \mid C}\right) .
$$

However, it is also known that there exists a pure state in $3 \otimes 2 \otimes 2$ quantum systems [9, 18],

$$
|\psi\rangle_{A B C}=\frac{1}{\sqrt{6}}(\sqrt{2}|010\rangle+\sqrt{2}|101\rangle+|200\rangle+|211\rangle),
$$

where $\tau\left(|\psi\rangle_{A \mid B C}\right)=\frac{12}{9}$ and $\tau\left(\rho_{A \mid B}\right)=\tau\left(\rho_{A \mid C}\right)=\frac{8}{9}$, therefore

$$
\tau\left(|\psi\rangle_{A \mid B C}\right)<\tau\left(\rho_{A \mid B}\right)+\tau\left(\rho_{A \mid C}\right) .
$$

In other words, the counterexample for three-party CKW inequality in Eq. (26) is also a counterexample for SM inequality in (22) in higher-dimensional quantum systems rather than qubits. Thus tangle-based SM inequality can only be valid for multi-qubit systems and even a tiny extension in any of the subsystems leads to a violation.

Here we propose another generalization of multi-qubit SM inequality into higher-dimensional quantum systems using SCREN. Due to the coincidence of tangle and SCREN for two-qubit states and any pure state of Schmidt-rank two in Eq. (16), the definition of threetangle in Eq. (18) can be naturally rephrased in terms of SCREN; for any three-qubit pure state $|\psi\rangle_{A B C}$,

$$
\begin{aligned}
\mathcal{N}_{s c}\left(|\psi\rangle_{A|B| C}\right)= & \mathcal{N}_{s c}\left(|\psi\rangle_{A \mid B C}\right) \\
& -\mathcal{N}_{s c}\left(\rho_{A \mid B}\right)-\mathcal{N}_{s c}\left(\rho_{A \mid C}\right) .
\end{aligned}
$$

For analogous terminology, we denote $\mathcal{N}_{s c}\left(|\psi\rangle_{A|B| C}\right)$ in Eq. (28) as three-SCREN where $\mathcal{N}_{s c}\left(|\psi\rangle_{A \mid B C}\right)$ and $\mathcal{N}_{s c}\left(\rho_{A \mid B}\right)$ are one- and two-SCREN, respectively.

Now we generalize the definition of three-SCREN in Eq. (28) into arbitrary multi-party, higher-dimensional quantum systems. For an $n$-qudit pure state $|\psi\rangle_{A_{1} A_{2} \cdots A_{n}}$, its $n-S C R E N$ is defined as 


$$
\mathcal{N}_{s c}\left(|\psi\rangle_{A_{1}\left|A_{2}\right| \cdots \mid A_{n}}\right)=\mathcal{N}_{s c}\left(|\psi\rangle_{A_{1} \mid A_{2} \cdots A_{n}}\right)-\sum_{m=2}^{n-1} \sum_{\vec{j}^{m}} \mathcal{N}_{s c}\left(\rho_{A_{1}\left|A_{j_{1}^{m}}\right| \cdots \mid A_{j_{m-1}^{m}}}\right)^{m / 2}
$$

where $\mathcal{N}_{s c}\left(|\psi\rangle_{A_{1} \mid A_{2} \cdots A_{n}}\right)$ is the one-SCREN of $n$-qudit pure state with respect to the bipartition between $A_{1}$ and the other qudit systems, and the $m$-SCREN of $m$-qubit reduced density matrix $\rho_{A_{1} A_{j_{1}^{m}} \cdots A_{j_{m-1}^{m}}}$ is defined as

$$
\mathcal{N}_{s c}\left(\rho_{A_{1}\left|A_{j_{1}^{m}}\right| \cdots \mid A_{j_{m-1}^{m}}}\right)=\left[\min _{\left\{p_{h},\left|\psi_{h}\right\rangle\right\}} \sum_{h} p_{h} \sqrt{\mathcal{N}_{s c}\left(\left|\psi_{h}\right\rangle_{A_{1}\left|A_{j_{1}^{m}}\right| \cdots \mid A_{j_{m-1}^{m}}}\right)}\right]^{2}
$$

with the minimization over all possible pure state decompositions of $\rho_{A_{1} A_{j_{1}^{m}} \cdots A_{j_{m-1}^{m}}}$. We also note that the index vector $\vec{j}^{m}=\left(j_{1}^{m}, \ldots, j_{m-1}^{m}\right)$ in the second summation of Eq. (29) spans all the ordered subsets of the index set $\{2, \ldots, n\}$ with $(m-1)$ distinct elements.

For a multi-qudit pure state $|\psi\rangle_{A_{1} A_{2} \cdots A_{n}}$, the $S C R E N$ $S M$ inequality of multi-party entanglement can be derived as

$\mathcal{N}_{s c}\left(|\psi\rangle_{A_{1} \mid A_{2} \cdots A_{n}}\right) \geq \sum_{m=2}^{n-1} \sum_{\vec{j}^{m}} \mathcal{N}_{s c}\left(\rho_{A_{1}\left|A_{j_{1}^{m}}\right| \cdots \mid A_{j_{m-1}^{m}}}\right)^{m / 2}$,

conjecturing the nonnegativity of $n$-SCREN in Eq. (29). From the relation of SCREN and tangle in Eq. (16), Inequality (31) is reduced to Inequality (22) for any multiqubit states. Thus Inequality (31) is a generalization of multi-qubit SM inequality in terms of tangle, which is valid for the classes of multi-qubit quantum states considered in [15, 16].

For the counterexample of CKW inequality in Eq. (26), it is straightforward to check $\mathcal{N}_{s c}\left(|\psi\rangle_{A \mid B C}\right)=4$ whereas $\mathcal{N}_{s c}\left(\rho_{A \mid B}\right)=\mathcal{N}_{s c}\left(\rho_{A \mid C}\right)=\frac{8}{9}$, and thus

$$
\mathcal{N}_{s c}\left(|\psi\rangle_{A \mid B C}\right) \geq \mathcal{N}_{s c}\left(\rho_{A \mid B}\right)+\mathcal{N}_{s c}\left(\rho_{A \mid C}\right) .
$$

Moreover, for the other counterexample in $3 \otimes 3 \otimes 3$ quantum systems [17,

$$
\begin{aligned}
|\psi\rangle_{A B C}=\frac{1}{\sqrt{6}} & (|123\rangle-|132\rangle+|231\rangle \\
& -|213\rangle+|312\rangle-|321\rangle),
\end{aligned}
$$

we have $\mathcal{N}_{s c}\left(|\psi\rangle_{A \mid B C}\right)=4$ whereas $\mathcal{N}_{s c}\left(\rho_{A \mid B}\right)=$ $\mathcal{N}_{s c}\left(\rho_{A \mid C}\right)=1$. In other words, Inequality (32) is still true for all the known counterexamples of CKW inequality, therefore SCREN is a good alternative of tangle in characterizing strongly monogamous property of multiparty entanglement.

\section{SCREN STRONG MONOGAMY INEQUALITY OF MULTI-QUDIT ENTANGLEMENT}

\section{A. Multi-Qudit Generalized W-class States}

Let us recall the definition of multi-qudit generalized W-class state [18],

$$
\begin{gathered}
\left|W_{n}^{d}\right\rangle_{A_{1} \cdots A_{n}}=\sum_{i=1}^{d-1}\left(a_{1 i}|i 0 \cdots 0\rangle+a_{2 i}|0 i \cdots 0\rangle\right. \\
\left.+\cdots+a_{n i}|00 \cdots 0 i\rangle\right),
\end{gathered}
$$

with the normalization condition $\sum_{s=1}^{n} \sum_{i=1}^{d-1}\left|a_{s i}\right|^{2}=1$. The state in Eq. (34) is a coherent superposition of all $n$ qudit product states with Hamming weight one. We also note that the term "generalized" naturally arises because Eq. (34) includes $n$-qubit W-class states in Eq. (24) as a special case when $d=2$.

Before we further investigate strongly monogamous property of entanglement for this generalized W-class state, we first recall a very useful property of quantum states proposed by Hughston-Jozsa-Wootters(HJW) showing the unitary freedom in the ensemble for density matrices [29].

Proposition 1. (HJW theorem) The sets $\left\{\left|\tilde{\phi}_{i}\right\rangle\right\}$ and $\left\{\left|\tilde{\psi}_{j}\right\rangle\right\}$ of (possibly unnormalized) states generate the same density matrix if and only if

$$
\left|\tilde{\phi}_{i}\right\rangle=\sum_{j} u_{i j}\left|\tilde{\psi}_{j}\right\rangle
$$

where $\left(u_{i j}\right)$ is a unitary matrix of complex numbers, with indices $i$ and $j$, and we pad whichever set of states $\left\{\left|\tilde{\phi}_{i}\right\rangle\right\}$ or $\left\{\left|\tilde{\psi}_{j}\right\rangle\right\}$ is smaller with additional zero vectors so that the two sets have the same number of elements.

A direct consequence of Proposition 11 is the following; for two pure-state decompositions $\sum_{i} p_{i}\left|\phi_{i}\right\rangle\left\langle\phi_{i}\right|$ and 
$\sum_{j} q_{j}\left|\psi_{j}\right\rangle\left\langle\psi_{j}\right|$, they represent the same density matrix, that is $\rho=\sum_{i} p_{i}\left|\phi_{i}\right\rangle\left\langle\phi_{i}\left|=\sum_{j} q_{j}\right| \psi_{j}\right\rangle\left\langle\psi_{j}\right|$ if and only if $\sqrt{p_{i}}\left|\phi_{i}\right\rangle=\sum_{j} u_{i j} \sqrt{q_{j}}\left|\psi_{j}\right\rangle$ for some unitary matrix $u_{i j}$. Using Proposition 1, we provide the following lemma, which shows a structural property of multi-qudit generalized W-class states.

Lemma 1. Let $|\psi\rangle_{A_{1} \ldots A_{n}}$ be a n-qudit pure state in a superposition of a $n$-qudit generalized $W$-class state in $E q$. (24) and vacuum, that is,

$$
|\psi\rangle_{A_{1} A_{2} \cdots A_{n}}=\sqrt{p}\left|W_{n}^{d}\right\rangle_{A_{1} \cdots A_{n}}+\sqrt{1-p}|0 \cdots 0\rangle_{A_{1} \cdots A_{n}}
$$

for $0 \leq p \leq 1$. Let $\rho_{A_{1} A_{j_{1}} \cdots A_{j_{m-1}}}$ be a reduced density matrix of $|\psi\rangle_{A_{1} \cdots A_{n}}$ onto m-qudit subsystems $A_{1} A_{j_{1}} \cdots A_{j_{m-1}}$ with $2 \leq m \leq n-1$. For any pure state decomposition of $\rho_{A_{1} A_{j_{1}} \cdots A_{j_{m-1}}}$ such that

$$
\rho_{A_{1} A_{j_{1}} \cdots A_{j_{m-1}}}=\sum_{k} q_{k}\left|\phi_{k}\right\rangle_{A_{1} A_{j_{1}} \cdots A_{j_{m-1}}}\left\langle\phi_{k}\right|,
$$

$\left|\phi_{k}\right\rangle_{A_{1} A_{j_{1}} \cdots A_{j_{m-1}}}$ is a superposition of a m-qudit generalized $W$-class state and vacuum.

Proof. Due to the symmetry of the structure of multiqudit generalized W-class states with respect to permuting subsystems, here we only consider the reduced density matrix $\rho_{A_{1} A_{2} \cdots A_{m}}$ of the first $m$ qudits subsystems $A_{1} A_{2} \cdots A_{m}$, where the general cases of $m$-qudit subsystems $A_{1} A_{j_{1}} \cdots A_{j_{m-1}}$ is then analogously following.

From a straightforward calculation, we obtain

$$
\rho_{A_{1} A_{2} \cdots A_{m}}=|\tilde{x}\rangle_{A_{1} A_{2} \cdots A_{m}}\langle\tilde{x}|+| \tilde{y}\rangle_{A_{1} A_{2} \cdots A_{m}}\langle\tilde{y}|,
$$

where

$$
\begin{aligned}
|\tilde{x}\rangle_{A_{1} A_{2} \cdots A_{m}}= & \sqrt{p} \sum_{i=1}^{d-1}\left(a_{1 i}|i 0 \cdots 0\rangle_{A_{1} A_{2} \cdots A_{m}}+a_{2 i}|0 i 0 \cdots 0\rangle_{A_{1} A_{2} \cdots A_{m}}+\cdots+a_{m i}|00 \cdots i\rangle_{A_{1} A_{2} \cdots A_{m}}\right) \\
& +\sqrt{1-p}|00 \cdots 0\rangle_{A_{1} A_{2} \cdots A_{m}}, \\
|\tilde{y}\rangle_{A_{1} A_{2} \cdots A_{m}}= & \sqrt{p \sum_{i=1}^{d-1}\left(\left|a_{m+1 i}\right|^{2}+\cdots+\left|a_{n i}\right|^{2}\right)|00 \cdots 0\rangle_{A_{1} A_{2} \cdots A_{m}}}
\end{aligned}
$$

are the unnormalized states in $m$-qubit subsystems $A_{1} A_{2} \cdots A_{m}$.

Now, let us consider the unnormalized states $\left|\tilde{\phi_{k}}\right\rangle_{A_{1} A_{2} \cdots A_{m}}=\sqrt{q_{k}}\left|\phi_{k}\right\rangle_{A_{1} A_{2} \cdots A_{m}}$ for each $k$ in the purestate decomposition Eq. (37). From Proposition 1, there exists an $r \times r$ unitary matrix $\left(u_{k l}\right)$ such that

$$
\left|\tilde{\phi_{k}}\right\rangle_{A_{1} A_{2} \cdots A_{m}}=u_{k 1}|\tilde{x}\rangle_{A_{1} A_{2} \cdots A_{m}}+u_{k 2}|\tilde{y}\rangle_{A_{1} A_{2} \cdots A_{m}}
$$

for each $k$. Moreover, Eqs. (39) imply that both $|\tilde{x}\rangle_{A_{1} A_{2} \cdots A_{m}}$ and $|\tilde{y}\rangle_{A_{1} A_{2} \cdots A_{m}}$ are linear combinations of $m$-qudit generalized W-class states and vacuums. In other words, $\left|\tilde{\phi_{k}}\right\rangle_{A_{1} A_{2} \cdots A_{m}}$ in Eq. (40) is an unnormalized superposition of a $m$-qudit generalized $\mathrm{W}$-class state and vacuum for each $k$. Thus the same is true for the normalized state $\left|\phi_{k}\right\rangle_{A_{1} A_{2} \cdots A_{m}}$ for each $k$.

\section{B. SCREN Strong Monogamy Inequality and Generalized W-class States}

In this scetion, we prove that the multi-qudit SCREN SM inequality of entanglement is true for a large class of multi-qubit quantum states in Eq. (36); superposition of multi-qudit generalized $\mathrm{W}$-class states and vacuums. We first provide the following theorem about the multiqudit generalized W-class and the CKW-type monogamy inequality.

Theorem 2. For a n-qudit pure state

$$
|\psi\rangle_{A_{1} A_{2} \cdots A_{n}}=\sqrt{p}\left|W_{n}^{d}\right\rangle_{A_{1} \cdots A_{n}}+\sqrt{1-p}|0 \cdots 0\rangle_{A_{1} \cdots A_{n}}
$$

where $\left|W_{n}^{d}\right\rangle_{A_{1} \cdots A_{n}}$ is a n-qudit generalized $W$-class state in Eq. 24) and $|0 \cdots 0\rangle_{A_{1} \cdots A_{n}}$ is the vacuum, we have

$\mathcal{N}_{s c}\left(|\psi\rangle_{A_{1} \mid A_{2} \cdots A_{n}}\right)=\mathcal{N}_{s c}\left(\rho_{A_{1} \mid A_{2}}\right)+\cdots+\mathcal{N}_{s c}\left(\rho_{A_{1} \mid A_{n}}\right)$,

where $\mathcal{N}_{s c}\left(|\psi\rangle_{A_{1} \mid A_{2} \cdots A_{n}}\right)$ is the ons-SCREN of $|\psi\rangle_{A_{1} A_{2} \cdots A_{n}}$ with respect to the bipartition between $A_{1}$ and the other qudits, and $\mathcal{N}_{s c}\left(\rho_{A_{1} \mid A_{s}}\right)$ is the twoSCREN of of the two-qudit state $\rho_{A_{1} A_{s}}$ with $s=2, \cdots, n$. 
Proof. For the one-SCREN of $|\psi\rangle_{A_{1} \cdots A_{n}}$ with respect to the bipartition between $A_{1}$ and the other qudits, the reduced density matrix $\rho_{A_{1}}$ of $|\psi\rangle_{A_{1} \cdots A_{n}}$ onto subsystem $A_{1}$ is obtained as

$$
\begin{aligned}
\rho_{A_{1}}= & \operatorname{tr}_{A_{2} \cdots A_{n}}|\psi\rangle_{A_{1} A_{2} \ldots A_{n}}\langle\psi| \\
= & p \sum_{i, j=1}^{d-1} a_{1 i} a_{1 j}^{*}|i\rangle_{A_{1}}\langle j|+[p \Omega+(1-p)]| 0\rangle_{A_{1}}\langle 0| \\
& +\sqrt{p(1-p)}\left[\sum_{i=1}^{d-1} a_{1 i}|i\rangle_{A_{1}}\left\langle 0\left|+\sum_{j=1}^{d-1} a_{1 j}^{*}\right| 0\right\rangle_{A_{1}}\langle j|\right],
\end{aligned}
$$

where $\Omega=\sum_{s=2}^{n} \sum_{i=1}^{d-1}\left|a_{s i}\right|^{2}=1-\sum_{j=1}^{d-1}\left|a_{1 j}\right|^{2}$.

From the the definition of pure state negativity in Eq. (4) together with Eq. (43), we have the one-SCREN of $|\psi\rangle_{A_{1} A_{2} \cdots A_{n}}$ between $A_{1}$ and the other qudits as

$$
\begin{aligned}
\mathcal{N}_{s c}\left(|\psi\rangle_{A_{1} \mid A_{2} \cdots A_{n}}\right) & =\left(\left(\operatorname{tr} \sqrt{\rho_{A_{1}}}\right)^{2}-1\right)^{2} \\
& =4 p^{2}(1-\Omega) \Omega .
\end{aligned}
$$

For the two-SCREN's $\mathcal{N}_{s c}\left(\rho_{A_{1} A_{s}}\right)$ with $s=2, \cdots, n$ that appear the right-hand side of Eq. (42), we first consider the case when $s=2$, where all the other cases are analogously following. The two-qudit reduced density matrix $\rho_{A_{1} A_{2}}$ of $|\psi\rangle_{A_{1} A_{2} \ldots A_{n}}$ is obtained as

$$
\begin{aligned}
\rho_{A_{1} A_{2}}= & \operatorname{tr}_{A_{3} \cdots A_{n}}|\psi\rangle_{A_{1} A_{2} \ldots A_{n}}\langle\psi| \\
= & p \sum_{i, j=1}^{d-1}\left[a_{1 i} a_{1 j}^{*}|i 0\rangle_{A_{1} A_{2}}\left\langle j 0\left|+a_{1 i} a_{2 j}^{*}\right| i 0\right\rangle_{A_{1} A_{2}}\left\langle 0 j\left|+a_{2 i} a_{1 j}^{*}\right| 0 i\right\rangle_{A_{1} A_{2}}\left\langle j 0\left|+a_{2 i} a_{2 j}^{*}\right| 0 i\right\rangle_{A_{1} A_{2}}\langle 0 j|\right] \\
& +\left(\Omega_{2}+1-p\right)|00\rangle_{A_{1} A_{2}}\langle 00| \\
& +\sqrt{p(1-p)} \sum_{k=1}^{d-1}\left[\left(a_{1 k}|k 0\rangle+a_{2 k}|0 k\rangle\right)_{A_{1} A_{2}}\left\langle 00\left|+a_{1 k}^{*}\right| 00\right\rangle_{A_{1} A_{2}}\left(\langle k 0|+a_{2 k}^{*}\langle 0 k|\right)\right]
\end{aligned}
$$

with $\Omega_{2}=1-\sum_{j=1}^{d-1}\left(\left|a_{1 j}\right|^{2}+\left|a_{2 j}\right|^{2}\right)$. We further note that, by considering two unnormalized states

$$
\begin{aligned}
|\tilde{x}\rangle_{A_{1} A_{2}} & =\sqrt{p} \sum_{i=1}^{d-1}\left(a_{1 i}|i 0\rangle_{A_{1} A_{2}}+a_{2 i}|0 i\rangle_{A_{1} A_{2}}\right) \\
& +\sqrt{1-p}|00\rangle_{A_{1} A_{2}}, \\
|\tilde{y}\rangle & =\sqrt{\Omega_{2}}|00\rangle_{A_{1} A_{2}},
\end{aligned}
$$

$\rho_{A_{1} A_{2}}$ in Eq. (45) can be represented as

$$
\rho_{A_{1} A_{2}}=|\tilde{x}\rangle_{A_{1} A_{2}}\langle\tilde{x}|+| \tilde{y}\rangle_{A_{1} A_{2}}\langle\tilde{y}| \text {. }
$$

Now Proposition 1 implies that for any pure state decomposition

$$
\rho_{A_{1} A_{2}}=\sum_{h}\left|\tilde{\phi_{h}}\right\rangle_{A_{1} A_{2}}\left\langle\tilde{\phi_{h}}\right|
$$

where $\left|\tilde{\phi_{h}}\right\rangle_{A_{1} A_{2}}$ is an unnormalized state in two-qudit subsystem $A_{1} A_{2}$, there exists an $r \times r$ unitary matrix $\left(u_{h l}\right)$ such that

$$
\left|\tilde{\phi}_{h}\right\rangle_{A_{1} A_{2}}=u_{h 1}|\tilde{x}\rangle_{A_{1} A_{2}}+u_{h 2}|\tilde{y}\rangle_{A_{1} A_{2}},
$$

for each $h$. For the normalized state $\left|\phi_{h}\right\rangle_{A_{1} A_{2}}=$ $\left|\tilde{\phi}_{h}\right\rangle_{A_{1} A_{2}} / \sqrt{p_{h}}$ with $p_{h}=\left|\left\langle\tilde{\phi}_{h} \mid \tilde{\phi}_{h}\right\rangle\right|$, the definition of pure state negativity in Eq. (4) leads us to the two-SCREN of
$\left|\phi_{h}\right\rangle_{A_{1} A_{2}}$

$$
\begin{aligned}
\mathcal{N}_{s c}\left(\left|\phi_{h}\right\rangle_{A_{1} \mid A_{2}}\right) & =\frac{4}{p_{h}^{2}} p^{2}\left|u_{h 2}\right|^{4}(1-\Omega)\left(\Omega-\Omega_{2}\right) \\
& =\frac{4}{p_{h}^{2}} p^{2}\left|u_{h 2}\right|^{4}(1-\Omega) \sum_{i=1}^{d-1}\left|a_{2 i}\right|^{2}
\end{aligned}
$$

for each $h$.

From the definition of SCREN for mixed states in Eq. (30) together with Eq. (50), we have the two-SCREN of $\rho_{A_{1} A_{2}}$ as

$$
\begin{aligned}
\mathcal{N}_{s c}\left(\rho_{A_{1} \mid A_{2}}\right) & =\left[\min _{\left\{p_{h},\left|\phi_{h}\right\rangle\right\}} \sum_{h} p_{h}{\sqrt{\mathcal{N}_{s c}\left(\left|\phi_{h}\right\rangle_{A_{1} \mid A_{2}}\right)}}^{2}\right. \\
& =\left[\min _{\left\{p_{h},\left|\phi_{h}\right\rangle\right\}} \sum_{h} 2 p\left|u_{h 2}\right|^{2}{\sqrt{(1-\Omega) \sum_{i=1}^{d-1}\left|a_{2 i}\right|^{2}}}^{2}\right. \\
& =4 p^{2}(1-\Omega) \sum_{i=1}^{d-1}\left|a_{2 i}\right|^{2}
\end{aligned}
$$

where the last equality is due to the choice of $u_{h 2}$ from the unitary matrix $\left(u_{h l}\right)$. Here we note that the minimum average of the square-root of SCREN in Eq. (51) does not depend on the choice of pure-state decomposition of $\rho_{A_{1} A_{2}}$, so that we could circumvent the minimization problem therein. 
By using an analogous method, we have the twoSCREN of two-qudit mixed state $\rho_{A_{1} A_{s}}$ as

$$
\mathcal{N}_{s c}\left(\rho_{A_{1} \mid A_{s}}\right)=4 p^{2}(1-\Omega) \sum_{i=1}^{d-1}\left|a_{s i}\right|^{2}
$$

for each $s=2, \cdots, n$. Now Eqs. (44) and (52) leads us to

$$
\begin{aligned}
\mathcal{N}_{s c}\left(|\psi\rangle_{A_{1} \mid A_{2} \cdots A_{n}}\right) & =4 p^{2}(1-\Omega) \Omega \\
& =4 p^{2}(1-\Omega) \sum_{s=2}^{n} \sum_{i=1}^{d-1}\left|a_{s i}\right|^{2} \\
& =\sum_{s=2}^{n}\left[4 p^{2}(1-\Omega) \sum_{i=1}^{d-1}\left|a_{s i}\right|^{2}\right] \\
& =\sum_{s=2}^{n} \mathcal{N}_{s c}\left(\rho_{A_{1} \mid A_{s}}\right),
\end{aligned}
$$

which completes the proof.

Theorem 2 implies that Inequality (17), the multiqubit CKW inequality in terms of one, and two-SCREN, is still true and in fact saturated for the class of multiqudit states in Eq. (36).

To check the validity of SCREN SM inequality in (31) for the class of states in Eq. (36), we first note that Inequality (31) can be decomposed as

$$
\begin{aligned}
\mathcal{N}_{s c}\left(|\psi\rangle_{A_{1} \mid A_{2} \cdots A_{n}}\right) \geq & \sum_{m=3}^{n-1} \sum_{\vec{j}^{m}} \mathcal{N}_{s c}\left(\rho_{A_{1}\left|A_{j_{1}^{m}}\right| \cdots \mid A_{j_{m-1}^{m}}}\right)^{m / 2} \\
& +\sum_{j=2}^{n} \mathcal{N}_{s c}\left(\rho_{A_{1} \mid A_{j}}\right)
\end{aligned}
$$

where the second summation of the first term on the right-hand side of the inequality runs over all the index vectors $\vec{j}^{m}=\left(j_{1}^{m}, \ldots, j_{m-1}^{m}\right)$ with $3 \leq m \leq n-1$.

By Theorem 2, the last term of the right-hand side and the left-hand side of of Inequality (54) are equal to each other for the class of states in Eq. (36). Thus this class of states are good candidates as possible counterexamples for stronger version of monogamy inequalities, that is, Inequality (31). Moreover, the validity of SCREN SM inequality for this class of states necessarily implies that Inequality (31) must be saturated, that is, the residual term

$$
\sum_{m=3}^{n-1} \sum_{\vec{j}^{m}} \mathcal{N}_{s c}\left(\rho_{A_{1}\left|A_{j_{1}^{m}}\right| \cdots \mid A_{j_{m-1}^{m}}}\right)^{m / 2}
$$

in (54) is zero for the class of states in Eq. (36). The following theorem states the main result of this paper, the saturation of multi-qudit SM inequality for the class of states in in Eq. (36).
Theorem 3. For the class of $n$-qudit states $|\psi\rangle_{A_{1} A_{2} \cdots A_{n}}$ in Eq. (36) that is a superposition of a $n$-qudit generalized $W$-class state and the vacuum, the multi-qudit SM inequality of entanglement in terms of SCREN is saturated;

$\mathcal{N}_{s c}\left(|\psi\rangle_{A_{1} \mid A_{2} \cdots A_{n}}\right)=\sum_{m=2}^{n-1} \sum_{\vec{j}^{m}} \mathcal{N}_{s c}\left(\rho_{A_{1}\left|A_{j_{1}^{m}}\right| \cdots \mid A_{j_{m-1}^{m}}}\right)^{m / 2}$.

Proof. As mentioned, it is enough to show that the residual term in Eq. (55) is zero for the class of states in Eq. (36). In fact, we further show that

$$
\mathcal{N}_{s c}\left(\rho_{A_{1}\left|A_{j_{1}^{m}}\right| \cdots \mid A_{j_{m-1}^{m}}}\right)=0
$$

for all the index vectors $\vec{j}^{m}=\left(j_{1}^{m}, \ldots, j_{m-1}^{m}\right)$ with $3 \leq$ $m \leq n-1$, that is, all the $m$-SCREN for $3 \leq m \leq$ $n-1$ is zero for the $m$-qudit reduced density matrices $\rho_{A_{1} A_{j_{1}^{m}} \cdots A_{j_{m-1}^{m}}}$.

We use the mathematical induction on $m$, and first consider the case when $m=3$. For any index vector $\vec{j}^{3}=\left(j_{1}, j_{2}\right)$ with $j_{1}, j_{2} \in\{2,3, \cdots, n\}$ [30], the lefthand side of Eq. (57) becomes the three-SCREN of the three-qudit reduced density matrix $\rho_{A_{1} A_{j_{1}} A_{j_{2}}}$,

$$
\begin{aligned}
& \mathcal{N}_{s c}\left(\rho_{A_{1}\left|A_{j_{1}}\right| A_{j_{2}}}\right) \\
& =\left[\min _{\left\{p_{h},\left|\psi_{h}\right\rangle\right\}} \sum_{h} p_{h} \sqrt{\mathcal{N}_{s c}\left(\left|\psi_{h}\right\rangle_{A_{1}\left|A_{j_{1}}\right| A_{j_{2}}}\right)}\right]^{2},
\end{aligned}
$$

where the minimization is over all possible pure state decompositions of $\rho_{A_{1} A_{j_{1}} A_{j_{2}}}$. Let us consider an optimal decomposition

$$
\rho_{A_{1} A_{j_{1}} A_{j_{2}}}=\sum_{k} q_{k}\left|\phi_{k}\right\rangle_{A_{1}\left|A_{j_{1}}\right| A_{j_{2}}}\left\langle\phi_{k}\right|,
$$

realizing the three-SCREN of of $\rho_{A_{1} A_{j_{1}} A_{j_{2}}}$,

$$
\begin{aligned}
& \mathcal{N}_{s c}\left(\rho_{A_{1}\left|A_{j_{1}}\right| A_{j_{2}}}\right) \\
&=\left[\sum_{k} q_{k} \sqrt{\mathcal{N}_{s c}\left(\left|\phi_{k}\right\rangle_{A_{1}\left|A_{j_{1}}\right| A_{j_{2}}}\right)}\right]^{2} .
\end{aligned}
$$

Because $\rho_{A_{1} A_{j_{1}} A_{j_{2}}}$ is a three-qudit reduced density matrix of $|\psi\rangle_{A_{1} A_{2} \cdots A_{n}}$ in Eq. (36), Lemma 1 implies that $\left|\phi_{k}\right\rangle_{A_{1} A_{j_{1}} A_{j_{2}}}$ in Eq. (59) is a superposition of a threequdit generalized $\mathrm{W}$-class state and vacuum for each $k$. Due to Theorem 2] we also note that CKW-type monogamy inequality in terms of SCREN is saturated by $\left|\phi_{k}\right\rangle_{A_{1} A_{j_{1}} A_{j_{2}}}$ in Eq. (59) for each $k$;

$$
\mathcal{N}_{s c}\left(\left|\phi_{k}\right\rangle_{A_{1} \mid A_{j_{1}} A_{j_{2}}}\right)=\mathcal{N}_{s c}\left(\rho_{A_{1} \mid A_{j_{1}}}^{k}\right)+\mathcal{N}_{s c}\left(\rho_{A_{1} \mid A_{j_{2}}}^{k}\right)
$$


where $\rho_{A_{1} A_{j_{1}}}^{k}$ and $\rho_{A_{1} A_{j_{2}}}^{k}$ are the reduced density matrices of $\left|\phi_{k}\right\rangle_{A_{1} A_{j_{1}} A_{j_{2}}}$ onto two-qudit subsystems $A_{1} A_{j_{1}}$ and $A_{1} A_{j_{2}}$ respectively.

From the definition of pure-state SCREN in Eq. (29) together with Eq. (61), we have

$$
\begin{aligned}
\mathcal{N}_{s c}\left(\left|\phi_{k}\right\rangle_{A_{1}\left|A_{j_{1}}\right| A_{j_{2}}}\right)= & \mathcal{N}_{s c}\left(\left|\phi_{k}\right\rangle_{A_{1} \mid A_{j_{1}} A_{j_{2}}}\right) \\
& -\mathcal{N}_{s c}\left(\rho_{A_{1} \mid A_{j_{1}}}^{k}\right)-\mathcal{N}_{s c}\left(\rho_{A_{1} \mid A_{j_{2}}}^{k}\right) \\
= & 0,
\end{aligned}
$$

for each three-qudit pure state $\left|\phi_{k}\right\rangle_{A_{1} \mid A_{j_{1}} A_{j_{2}}}$ in Eq. (60), and thus we have

$$
\mathcal{N}_{s c}\left(\rho_{A_{1}\left|A_{j_{1}}\right| A_{j_{2}}}\right)=0
$$

for any three-qudit reduced density matrix $\rho_{A_{1} A_{j_{1}} A_{j_{2}}}$ of $|\psi\rangle_{A_{1} A_{2} \cdots A_{n}}$ in Eq. (36).

Now we assume the induction hypothesis; for any $(m-$ 1 )-qudit reduced density matrix $\rho_{A_{1} A_{j_{1}} A_{j_{2}} \cdots A_{j_{m-2}}}$ of the state $|\psi\rangle_{A_{1} A_{2} \cdots A_{n}}$ in Eq. (36), we assume

$$
\mathcal{N}_{s c}\left(\rho_{A_{1}\left|A_{j_{1}}\right| A_{j_{2}}|\cdots| A_{j_{m-2}}}\right)=0 .
$$

For any index vector $\vec{j}=\left(j_{1}, j_{2}, \ldots, j_{m-1}\right)$ with $\left\{j_{1}, j_{2}, \ldots, j_{m-1}\right\} \subseteq\{2,3, \cdots, n\}$ and the $m$-qudit reduced density matrix $\rho_{A_{1} A_{j_{1}} \cdots A_{j_{m-1}}}$, we consider an optimal pure-state decomposition

$$
\rho_{A_{1} A_{j_{1}} \cdots A_{j_{m-1}}}=\sum_{k} q_{k}\left|\phi_{k}\right\rangle_{A_{1} A_{j_{1}} \cdots A_{j_{m-1}}}\left\langle\phi_{k}\right|
$$

realizing $m$-SCREN of $\rho_{A_{1} A_{j_{1}} \cdots A_{j_{m-1}}}$, that is,

$$
\begin{aligned}
& \mathcal{N}_{s c}\left(\rho_{A_{1}\left|A_{j_{1}}\right| \cdots \mid A_{j_{m-1}}}\right) \\
&=\left[\sum_{k} q_{k} \sqrt{\mathcal{N}_{s c}\left(\left|\phi_{k}\right\rangle_{A_{1}\left|A_{j_{1}}\right| \cdots \mid A_{j_{m-1}}}\right)}\right]^{2} .
\end{aligned}
$$

From the definition of pure-state SCREN in Eq. (29), the $m$-SCREN of each $\left|\phi_{k}\right\rangle_{A_{1} A_{j_{1}} \cdots A_{j_{m-1}}}$ in Eq. (66) is

$$
\begin{array}{r}
\mathcal{N}_{s c}\left(\left|\phi_{k}\right\rangle_{A_{1}\left|A_{j_{1}}\right| \cdots \mid A_{j_{m-1}}}\right)=\mathcal{N}_{s c}\left(\left|\phi_{k}\right\rangle_{A_{1} \mid A_{j_{1}} \cdots A_{j_{m-1}}}\right) \\
-\sum_{s=2}^{m-1} \sum_{\vec{i}^{s}} \mathcal{N}_{s c}\left(\rho_{A_{1}\left|A_{i_{1}}\right| \cdots \mid A_{i_{s-1}}}^{k}\right)^{s / 2},
\end{array}
$$

where $\rho_{A_{1} A_{i_{1}} \cdots A_{i_{s-1}}}^{k}$ is the reduced density matrix of $\left|\phi_{k}\right\rangle_{A_{1} A_{j_{1}} \cdots A_{j_{m-1}}}$ on $s$-qudit subsystems $A_{1} A_{i_{1}} \cdots A_{i_{s-1}}$, and the second summation is over all possible index vectors $\vec{i}^{s}=\left(i_{1}, i_{2}, \cdots, i_{s-1}\right)$ with $\left\{i_{1}, i_{2}, \cdots, i_{s-1}\right\} \subseteq$ $\left\{j_{1}, j_{2}, \cdots, j_{m-1}\right\}$. We further divide the last term of the right-hand side of Eq. (67) into the summations of two-SCREN and the others;

$$
\begin{array}{r}
\mathcal{N}_{s c}\left(\left|\phi_{k}\right\rangle_{A_{1}\left|A_{j_{1}}\right| \cdots \mid A_{j_{m-1}}}\right)=\mathcal{N}_{s c}\left(\left|\phi_{k}\right\rangle_{A_{1} \mid A_{j_{1}} \cdots A_{j_{m-1}}}\right) \\
-\sum_{l=1}^{m-1} \mathcal{N}_{s c}\left(\rho_{A_{1} \mid A_{j_{l}}}^{k}\right) \\
-\sum_{s=3}^{m-1} \sum_{\vec{i}^{s}} \mathcal{N}_{s c}\left(\rho_{A_{1}\left|A_{i_{1}}\right| \cdots \mid A_{i_{s-1}}}^{k}\right)^{s / 2} .
\end{array}
$$

For each $s=3, \cdots, m-1, \rho_{A_{1} A_{i_{1}} \cdots A_{i_{s-1}}}^{k}$ in the last summation of Eq. (68) is a $s$-qudit reduced density matrix of the $m$-qudit state $\left|\phi_{k}\right\rangle_{A_{1} A_{j_{1}} \cdots A_{j_{m-1}}}$ where Lemma 1 implies that $\left|\phi_{k}\right\rangle_{A_{1} A_{j_{1}} \cdots A_{j_{m-1}}}$ in Eq. (65) is a superposition of a $m$-qudit $\mathrm{W}$-class state and vacuum. Thus the induction hypothesis assures that the $s$-SCREN of $\rho_{A_{1} A_{i_{1}} \cdots A_{i_{s-1}}}^{k}$ is zero;

$$
\mathcal{N}_{s c}\left(\rho_{A_{1}\left|A_{i_{1}}\right| \cdots \mid A_{i_{s-1}}}^{k}\right)=0,
$$

for each $s=3, \cdots, m-1$ and the index vector $\vec{i}^{s}=$ $\left(i_{1}, i_{2}, \cdots, i_{s-1}\right)$.

Furthermore, Theorem 2 implies that the CKW-type monogamy inequality in terms of one and two SCREN is saturated by $\left|\phi_{k}\right\rangle_{A_{1} A_{j_{1}} \cdots A_{j_{m-1}}}$, that is,

$$
\mathcal{N}_{s c}\left(\left|\phi_{k}\right\rangle_{A_{1} \mid A_{j_{1}} \cdots A_{j_{m-1}}}\right)=\sum_{l=1}^{m-1} \mathcal{N}_{s c}\left(\rho_{A_{1} \mid A_{j_{l}}}^{k}\right),
$$

for each $k$. From Eq. (68) together with Eqs. (69) and (70), we have

$$
\mathcal{N}_{s c}\left(\left|\phi_{k}\right\rangle_{A_{1}\left|A_{j_{1}}\right| \cdots \mid A_{j_{m-1}}}\right)=0
$$

for each $\left|\phi_{k}\right\rangle_{A_{1} A_{j_{1}} \cdots A_{j_{m-1}}}$ that arises in the decomposition of $\rho_{A_{1} A_{j_{1}} \cdots A_{j_{m-1}}}$ in Eq. (65). Thus Eqs. (66) and (171) lead us to

$$
\mathcal{N}_{s c}\left(\rho_{A_{1}\left|A_{j_{1}}\right| \cdots \mid A_{j_{m-1}}}\right)=0
$$

for any the $m$-qudit reduced density matrix $\rho_{A_{1} A_{j_{1}} \cdots A_{j_{m-1}}}$ of $|\psi\rangle_{A_{1} A_{2} \ldots A_{n}}$ with $3 \leq m \leq n-1$.

\section{CONCLUSIONS}

In this paper, we have proposed SCREN as a powerful candidate to characterize the strongly monogamous property of multi-qudit systems. We have provided a SM inequality of multi-party entanglement in terms of SCREN, and shown that the tangle-based SM inequality of multi-qubit systems can be rephrased by SCREN. We 
have also shown that SCREN SM inequality is still true for the counterexamples of CKW inequality in higherdimensional systems. We have further provided an analytical proof that SCREN SM inequality is saturated by a large class of multi-qudit states, a superposition of multiqudit generalized $\mathrm{W}$-class states and vacuums. Thus SCREN is a good alternative of tangle in characterizing strong monogamy of multi-party entanglement without any known counterexample even in higher-dimensional systems.

Noting the importance of the study on multi-party quantum entanglement, our result can provide a rich ref- erence for future work on the study of entanglement in complex quantum systems.

\section{Acknowledgments}

This research was supported by Basic Science Research Program through the National Research Foundation of Korea(NRF) funded by the Ministry of Education, Science and Technology(NRF-2014R1A1A2056678) and Fusion Technology R\&D Center of SK Telecom.
[1] C. H. Bennett, G. Brassard, C. Crepeau, R. Jozsa, A. Peres and W. K. Wootters, Phys. Rev. Lett. 70, 1895 (1993).

[2] C. Bennett and G. Brassard, in Proceedings of IEEE International Conference on Computers, Systems, and Signal Processing (IEEE Press, New York, Bangalore, India, 1984), p. 175-179.

[3] C. H. Bennett, Physical Review Letters 68, 3121 (1992).

[4] V. Coffman, J. Kundu and W. K. Wootters, Phys. Rev. A 61, 052306 (2000).

[5] T. Osborne and F. Verstraete, Phys. Rev. Lett. 96, 220503 (2006).

[6] M. Koashi and A. Winter, Phys. Rev. A 69, 022309 (2004)

[7] B. M. Terhal, IBM J. Research and Development 48, 71 (2004).

[8] J. S. Kim, G. Gour and B. C. Sanders, Contemp. Phys. 53, 5 p. 417-432 (2012).

[9] J. S. Kim, A. Das and B. C. Sanders, Phys. Rev. A 79, 012329 (2009).

[10] J. S. Kim and B. C. Sanders, J. Phys. A: Math. and Theor. 43, 445305 (2010).

[11] J. S. Kim, Phys. Rev. A. 81, 062328 (2010).

[12] J. S. Kim and B. C. Sanders, J. Phys. A: Math. and Theor. 44, 295303 (2011).

[13] M. Christandl and A. Winter, J. Math. Phys. 45, p. 829840 (2004).

[14] F. G. S. L. Brandao, M. Christandl and J. Yard, Commun. Math. Phys. 306, 805 (2011).

[15] B. Regula, S. D. Martino, S. Lee and G. Adesso, Phys. Rev. Lett. 113, 110501 (2014).

[16] J. S. Kim, Phys. Rev. A 90, 062306 (2014).

[17] Y. Ou, Phys. Rev. A 75, 034305 (2007).

[18] J. S. Kim and B. C. Sanders, J. Phys. A 41, 495301 (2008).

[19] G. Vidal and R. F. Werner, Phys. Rev. A 65, 032314
(2002).

[20] The definition of negativity for a state $\rho$ was first proposed by Vidal and Werner [19] as $\frac{\left\|\rho^{T} B\right\|_{1}-1}{2}$, and this corresponds to the absolute value of the sum of negative eigenvalues of $\rho^{T_{B}}$. Another definition of the negativity with a normalizing factor, $\frac{\left\|\rho^{T} B\right\|_{1}-1}{d-1}$, was also used for the states in a $d \otimes d^{\prime}\left(d \leq d^{\prime}\right)$ quantum system [26]. To avoid this inconsistency, here we only use $\left\|\rho^{T_{B}}\right\|_{1}-1$ for our definition of the negativity, which also coincides with the square-root of tangle for two-qubit pure states.

[21] A. Peres, Phys. Rev. Lett. 77, 1413 (1996).

[22] M. Horodecki, P. Horodecki and R. Horodecki, Phys. Lett. A 223, 1 (1996).

[23] P. Horodecki, Phys. Lett. A 232, 333 (1997).

[24] W. Dür, J.I. Cirac, M. Lewenstein and D. Bruß, Phys. Rev. A 61, 062313 (2000).

[25] M. Horodecki, P. Horodecki and R. Horodecki, Phys. Rev. Lett. 80, 5239 (1998).

[26] S. Lee, D. P. Chi, S. D. Oh and J. Kim, Phys, Rev. A 68, 62304 (2003).

[27] This definition is also valid for a bipartite pure state with Schmidt-rank 2, that is,

$$
|\psi\rangle_{A B}=\sqrt{\lambda_{1}}\left|e_{0}\right\rangle_{A} \otimes\left|f_{0}\right\rangle_{B}+\sqrt{\lambda_{2}}\left|e_{1}\right\rangle_{A} \otimes\left|f_{1}\right\rangle_{B}
$$

for some orthonormal bases $\left\{\left|e_{0}\right\rangle_{A},\left|e_{1}\right\rangle_{A}\right\}$ and $\left\{\left|f_{0}\right\rangle_{A},\left|f_{1}\right\rangle_{A}\right\}$ of subsystems $A$ and $B$, respectively.

[28] W. Dür, G. Vidal, and J. I. Cirac, Phys. Rev. A 62, 062314 (2000).

[29] L. P. Hughston, R. Jozsa and W. K. Wootters, Phys. Lett. A 183, 14 (1993).

[30] For simplicity, we sometimes omit the superscript $m$ in the components of the index vector $\vec{j}^{m}=\left(j_{1}^{m}, \ldots, j_{m-1}^{m}\right)$ if there is no confusion. 